\title{
Onsite Computed Tomography Fractional Flow Reserve in Patients with Suspected Stable Coronary Artery Disease Initial Experience
}

\author{
Atul Kapoor ${ }^{1}$ Goldaa Mahajan ${ }^{1}$ Aprajita Kapoor ${ }^{1}$ \\ ${ }^{1}$ Department of Computed Tomography, Advanced Diagnostics and \\ Institute of Imaging, Amritsar, Punjab, India \\ Indian J Radiol Imaging 2021;31:291-296.
}

Address for correspondence Atul Kapoor, 17/8 Kennedy Avenue, Amritsar 143001, Punjab, India (e-mail: masatulak@aim.com).

\begin{abstract}
Keywords

- computed tomographic coronary angiography

- coronary artery disease

- CTFFR
\end{abstract}

Introduction Computed tomographic coronary angiographic (CTCA) has evolved into a robust technique to detect significant stenosis; however, there is a discordance in the anatomic and functional significance of stenosis. Therefore, patients with stable coronary artery disease need to be further evaluated before deciding for any revascularization procedure.

Material and Methods A total of 100 consecutive patients of suspected stable coronary artery disease who underwent CTCA were evaluated for functional significance of lesions using onsite computed tomography fractional flow reserve (CTFFR) and the results were compared for detection of both significant and hemodynamically significant/severe stenosis on per vessel and per patient basis and differences were statistically analyzed. Impact of these differences were analyzed for the final outcome and management plan.

Results CTCA detected 33 patients with severe stenosis, while 54 patients had hemodynamically significant stenosis on CTFFR. The sensitivity and specificity of CTCA for the detection of significant coronary artery stenosis per vessel basis were 97.7 and $93.3 \%$, respectively, with a negative predictive value of $98.0 \%$. For severe coronary artery stenosis, sensitivity, specificity, and negative and positive predictive values were $51.6,89,68.8$, and $80 \%$, respectively, on per vessel basis with CTCA. On per patient basis, CTCA showed as sensitivity and specificity of 61 and $84 \%$, respectively, with area under curve (AUC) being 0.92 and 0.67 for significant and severe stenosis, respectively.

Discussion Onsite CTFFR is a useful tool to calculate functionally significant stenosis and also improves the sensitivity and specificity of CTCA. CTFFR detected $12 \%$ more stenotic vessels in the present study on per vessel basis and $21 \%$ on per patient basis.

\section{Introduction}

Advances in the technology of computed tomographic coronary angiography (CTCA) have led to high sensitivity for

published online July 28, 2021
DOI https://doi.org/

$10.1055 / \mathrm{s}-0041-1734363$ ISSN 0971-3026 identification of the disease. Zhang et al $^{1}$ showed sensitivity and specificity on per-patient basis of 93.9 and $93.5 \%$, respectively, for significant stenosis for CTCA. The National Institute for Health and Care Excellence (NICE) 2016 guidelines (c) 2021. Indian Radiological Association.

This is an open access article published by Thieme under the terms of the Creative Commons Attribution-NonDerivative-NonCommercial-License, permitting copying and reproduction so long as the original work is given appropriate credit. Contents may not be used for commercial purposes, or adapted, remixed, transformed or built upon. (https://creativecommons.org/licenses/by-nc-nd/4.0/). Thieme Medical and Scientific Publishers Private Ltd. A-12, Second Floor, Sector -2, NOIDA -201301, India 
also recommend CTCA as the first line of investigation for evaluation of patients with typical or atypical angina who are stable and have electrocardiography (EKG) changes of ischemia. $^{2}$ Recently concluded PROMISE and SCOT trials ${ }^{3,4}$ have also concluded that use of CTCA is better noninvasive diagnostic tool than other noninvasive testing modalities in such patients and helps to reduce future major cardiac events. However, CTCA has also been shown to have a low positive predictive value. ${ }^{5}$ Therefore, it is recommended that patients having anatomically significant coronary artery disease with stenosis $>50 \%$ on CTCA should undergo another test for demonstrable ischemia before revascularization is considered. Invasive FFR was introduced by Pijls et al in $1996^{6}$ for the above purpose to determine the hemodynamic significance of stenosis and FAME trial ${ }^{7}$ has proven its value in the detection of clinical significance of lesions which need to be revascularized. Despite the evidence, less than $10 \%$ cardiac laboratories do this procedure. The flip side being that it is invasive and adds significant cost and procedure time. ${ }^{8}$ Noninvasive FFR using CTCA has evolved since 2019 and uses CTCA images to compute pressure based on the model of fluid dynamics and now with the use of machine learning algorithms, it is possible to get onsite computed tomography fractional flow reserve (CTFFR). ${ }^{9,10}$ This study was, therefore, designed to evaluate the role of onsite CTFFR in patients with suspected stable coronary artery disease who underwent CTCA examination in Indian population and clinical cardiology practice.

\section{Materials and Methods}

After approval from local ethical review committee, 100 consecutive patients of clinically suspected stable coronary artery disease were enrolled since January 2019 to December 2019. Informed consent was obtained from all the patients. The demographic parameters of all patients, that is, age, sex, body mass index (BMI) along with history of disease, coexisting morbidities like hypertension, diabetes, and hyperlipidemias were recorded. All patients with history of allergy to iodine, arrhythmias, heart rate of more than 100 beats/min, BMI more than $31 \mathrm{~kg} / \mathrm{m}^{2}$, increased creatinine levels $>1.2 \mathrm{mg} / \mathrm{mL}$, or any prior bypass grafting/angioplasty were excluded from the study. Calcium scoring was not done purposely so that calcified vessels may also be evaluated by CTFFR. CT examination was done on a dual-energy CT 128 scanner (Siemens Go-Top, Forchheim, Germany). Oral 50-mg metoprolol 1 hour before the examination was given followed by sublingual nitroglycerin $0.5 \mathrm{mg}$ to all patients except who had any history of hypotension. A retrospectively gated ECG triggered spiral (pitch 0.3) acquisition was done with collimation of $0.31 \mathrm{~mm}$ and gantry rotation time of $0.31 \mathrm{~ms}$ at a tube voltage of $70 \mathrm{KV}$ along with automated tube current modulation using CARE Dose $4 \mathrm{D}$, Siemens healthineers. The quality reference voltage and current was set to $120 \mathrm{kV}, 320 \mathrm{mAs}$. R-R interval was fixed at 35 to 75\%. Noniodinated contrast iomeprol 400 (Iomeron Bracco UK Ltd.) of $35 \mathrm{~mL}$ was injected using bolus tracking software with dual head injector (MEDRAD, Stellant, Bayers, Munich, Germany) with flow rate of $4 \mathrm{~mL} / \mathrm{s}$ followed by
$20 \mathrm{~mL}$ saline. Image reconstruction was done using iterative reconstruction (SAFIRE, Siemens healthineers level 3) with reconstruction kernel of BV36. All images were transferred to Siemens Syngo. via workstation for post processing in multi planar and volume rendering projections and read by second author (G.M.) who was blinded to the results of CTFFR done onsite on Siemens Syngovia Frontier workstation using CTFFR (version 2.0) software and the results read by first author (A.K.). CTFFR was displayed in the form of color maps of coronary tree with blue being normal and red being ischemic (-Fig. 1). Quantitative analysis was done by marking the analysis site at any point along the vessel. The degree of stenosis was recorded based on per vessel and per patient basis. Vessels with stenosis more than $50 \%$ were labeled as significant and more than $70 \%$ as severe stenosis. On CTFFR, vessels showing FFR below 0.8 distal to the stenotic site were taken as hemodynamically significant. The results of the impact of CTCA and CTFFR findings on the management plan were obtained from the clinicians in the form of questionnaire, that is, (1) any change in optimal medical management after CTCA and CTFFR and (2) change to intervention planelective angioplasty or surgery.

\section{Statistical Analysis}

Statistical analysis was done using Analyze-IT software (Leeds, United Kingdom). Distribution and confidence intervals of all continuous variables were done along with Shapiro-Wilk $W$-test of normality of distribution. Sensitivity and specificity of both CTCA and CTCA with CTFFR were calculated for per vessel and per patient basis along with area under the curve (AUC) analysis. $p$-Value was fixed at $<0.05$ as statistically significant with post hoc power of the test at 0.88 .

The primary objectives of the study were to determine (1) if on site CTFFR can be computed on images acquired using CTCA, (2) If CTFFR does identify hemodynamically significant stenosis $(<0.8)$, and (3) correlation of anatomic site with

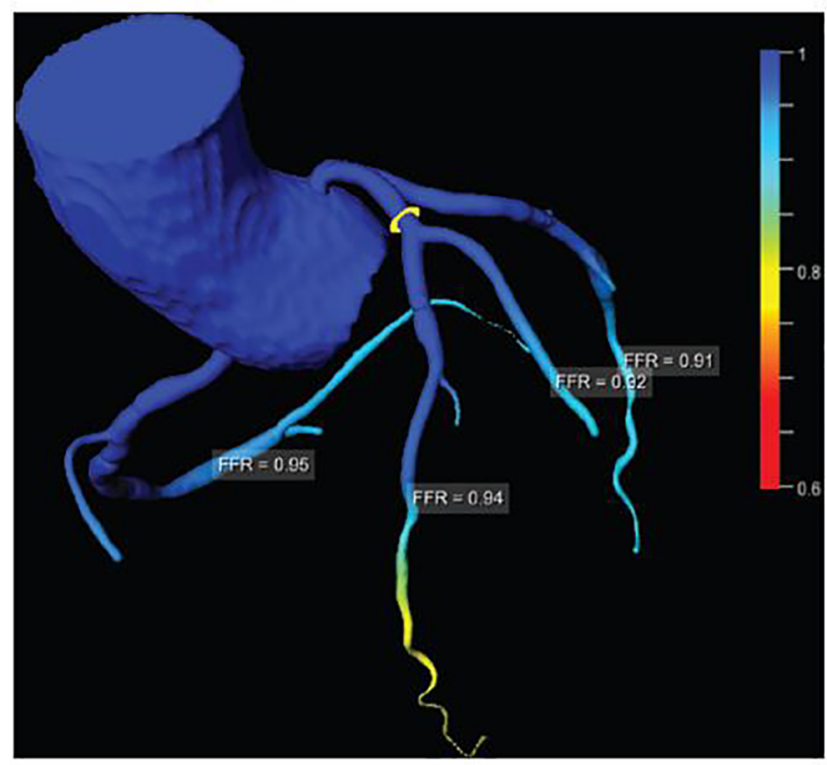

Fig. 1 CTFFR coronary map showing normal FFR in the coronary vasculature. CTFFR, computed tomography fractional flow reserve. 
functionally significant stenosis. Secondary objective was to find the impact of CTFFR on the management of patients with stable coronary artery disease.

\section{Results}

Demographics of the patients in the study are listed in - Table 1. Mean age of patients was 57 years. There were 74 males and 26 females. Mean heart rate was 72 beats $/ \mathrm{min}$. Significant stenosis was observed in 33 patients on per patient basis on CTCA with 125 vessels showing stenosis more than $50 \%$ while severe stenosis was seen in all 33 patients on per patient basis and in 72 vessels on per vessel basis. Distribution of stenotic lesions per vessels seen on CTCA were: left anterior descending 66 vessels of which 31 were severely stenotic and 22 had intermediate stenosis, right coronary artery showed stenosis in 48 vessels of which 19 were severely stenotic and 17 had intermediate stenosis, and 23 left circumflex arteries had significant stenosis with 9 being severely stenotic. Left main coronary artery showed 13 vessels with stenosis more than $50 \%$ all of which were detected by CTFFR ( $~-$ Fig. 2). Out of these, eight vessels showed calcified plaques, while five were noncalcified ( - Figs. $\mathbf{3 A}$ and B ). Comparatively, CTFFR detected hemodynamically significant stenosis in 128 vessels, that is, FFR $<0.8$ in 54 patients

Table 1 Patient demographics

\begin{tabular}{|l|l|l|l|}
\hline Sl. no & Variable name & Mean & $95 \% \mathrm{Cl}$ \\
\hline 1 & Mean age (y) & 57 & $54.8-59.2$ \\
\hline 2 & Sex & & \\
\cline { 2 - 4 } & Males & 74 & \\
\cline { 2 - 4 } & Females & 26 & $26.6-30.6$ \\
\hline 3 & BMI $\left(\mathrm{kg} / \mathrm{m}^{2}\right)$ & 30.16 & \\
\hline 4 & Hypertension & 80 & \\
\hline 5 & Diabetes & 45 & \\
\hline 6 & Dyslipidemia & 65 & \\
\hline 7 & Hyperuricemia & 21 & \\
\hline 8 & $\begin{array}{l}\text { History of antianginal } \\
\text { drugs }\end{array}$ & 28 & $64-85$ \\
\hline 9 & History of oral statins & 44 & 72 beats/min \\
\hline 10 & Mean heart rate & & \\
\hline
\end{tabular}

Abbreviations: BMI, body mass index; $\mathrm{Cl}$, confidence interval.

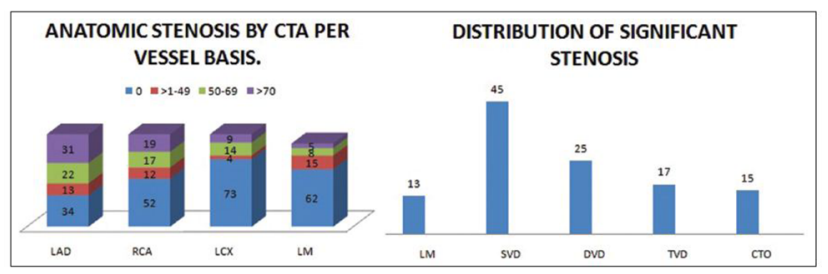

Fig. 2 Bar charts showing distribution of anatomic stenosis per vessel basis and distribution of significant stenosis by CTCA. CTA, computed tomography angiography; CTCA, computed tomographic coronary angiography.

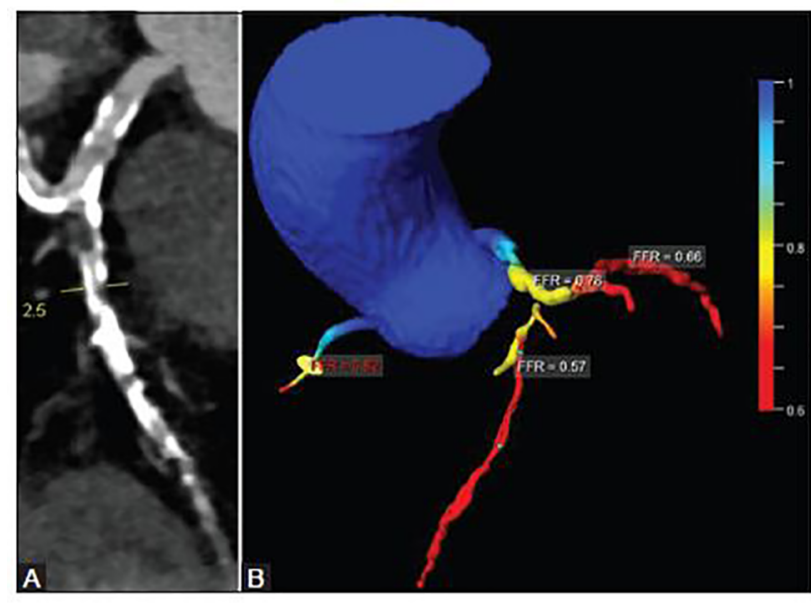

Fig. 3 (A) CTCA showing calcified plaque with bifurcation stenosis of left main coronary artery and proximal left anterior descending artery. (B) CTFFR showing reduced distal FFR in the same patient. CTCA, computed tomographic coronary angiography; CTFFR, computed tomography fractional flow reserve.

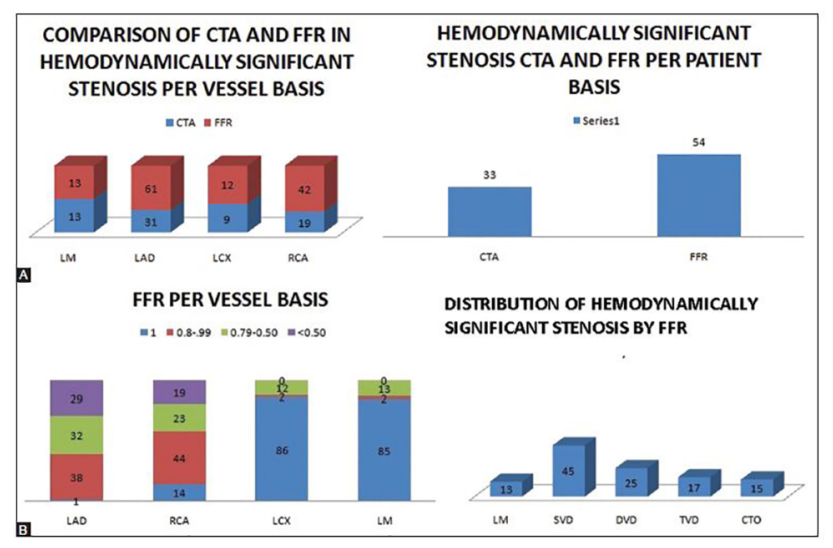

Fig. 4 (A) Bar charts showing comparison of CTCA and FFR in hemodynamically significant stenosis on per vessel and per patient basis. (B): Bar charts showing distribution of lesions by FFR on per vessel basis and in hemodynamically significant stenosis. CTA, computed tomography angiography; CTCA, computed tomographic coronary angiography; FFR, fractional flow reserve.

details enlisted in $\boldsymbol{- F i g . ~ 4 A}$. Reduced FFR of $<0.8$ was seen in 61 left anterior descending arteries, 32 right coronary arteries, 12 left circumflex arteries, and 13 left main coronaries these being hemodynamically significant stenosis ( - Figs. 4B and 5A-D). There were 18 coronary vessels on CTCA which were false positive as having significant stenosis but had normal FFR ( - Figs. 6A and B ). A total of 56 vessels were labeled as having intermediate stenosis, that is, 50 to $69 \%$ had FFR of $<0.8$ which leads to $21 \%$ difference in severity of stenosis on per patient basis ( - Figs. 7A and B ), The sensitivity and specificity of CTCA for detection of significant coronary artery stenosis per vessel basis were 97.7 and $93.3 \%$ with a negative predictive value of $98.0 \%$. For severe coronary artery stenosis, CTCA showed a sensitivity, specificity, and negative and positive predictive values of $51.6,89,68.8$, and $80 \%$, respectively, on per vessel basis ( $\boldsymbol{- \text { Fig. }}$ 8A). On per patient basis, CTCA showed as sensitivity and specificity of 61 and 


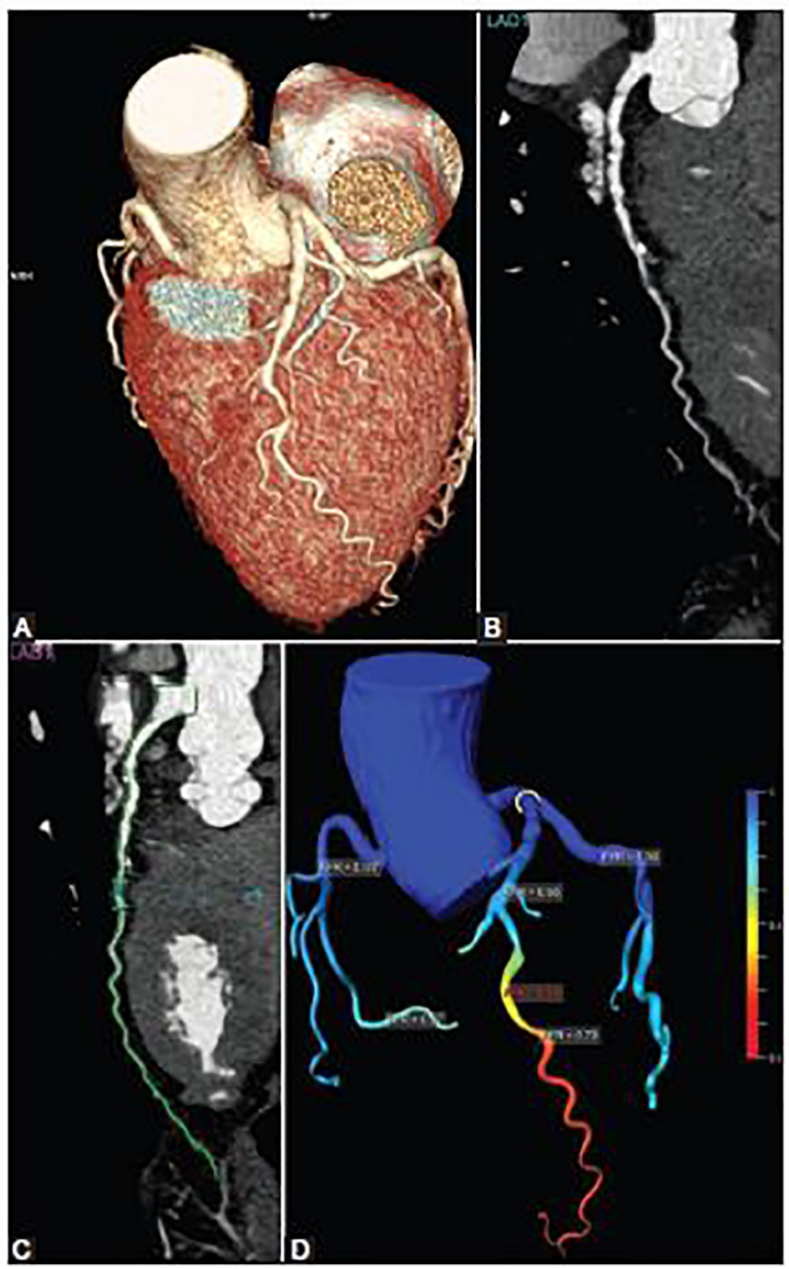

Fig. 5 (A) VRT image showing multiple areas of stenosis in left anterior descending artery. (B) Multiplanar view of the left anterior descending coronary artery with multiple calcified and soft plaques with indeterminate site of significant stenosis. (C) Multiplanar view of the same patient with boundary delineations by CTFFR. (D) FFR color map showing hemodynamic significant stenosis in the same patient with triple vessel disease. CTFFR, computed tomography fractional flow reserve.

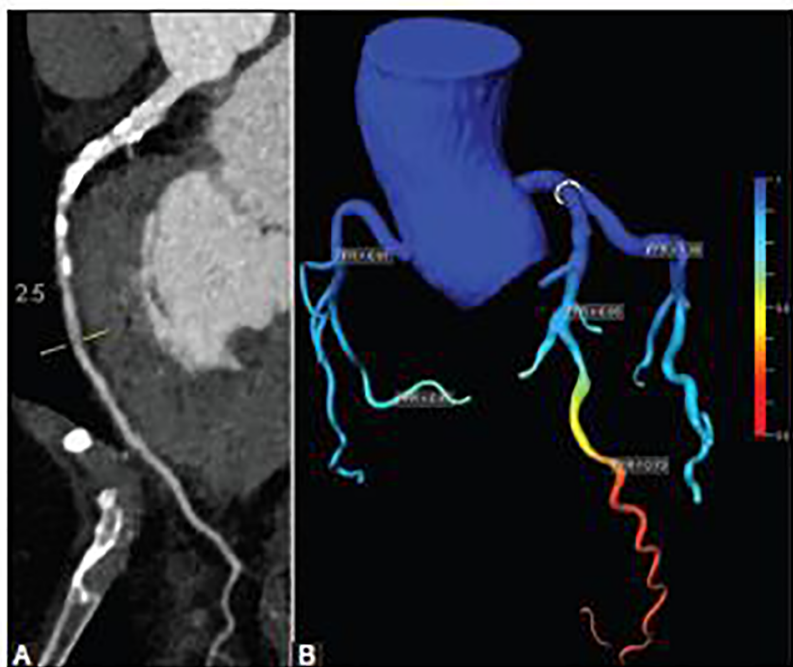

Fig. 6 (A) CT angiogram multiplanar reconstruction showing multiple calcified plaques with high-grade stenosis in proximal left anterior descending artery. (B) CTFFR showing no hemodynamically significant stenotic lesion. CTFFR, computed tomography fractional flow reserve.
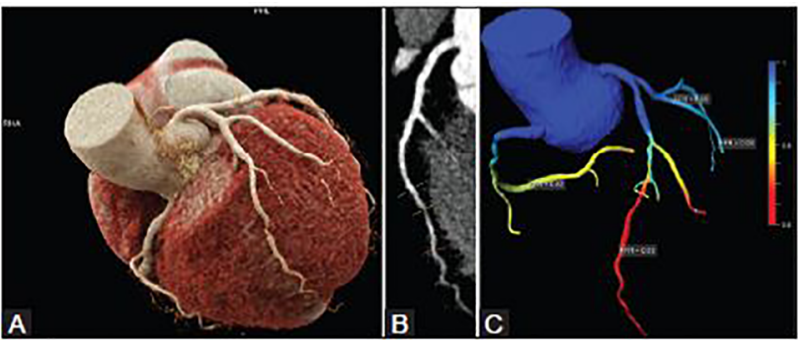

Fig. 7 (A) VR 3D image on CT angiogram showing stenosis in mid left anterior descending artery. (B) Multiplanar reconstruction image of the same vessel showing $65 \%$ stenosis in mid segment. (C) CTFFR showing reduced FFR distal to the stenosis in mid segment in the same patient. 3D, three-dimensional; CTFFR, computed tomography fractional flow reserve.

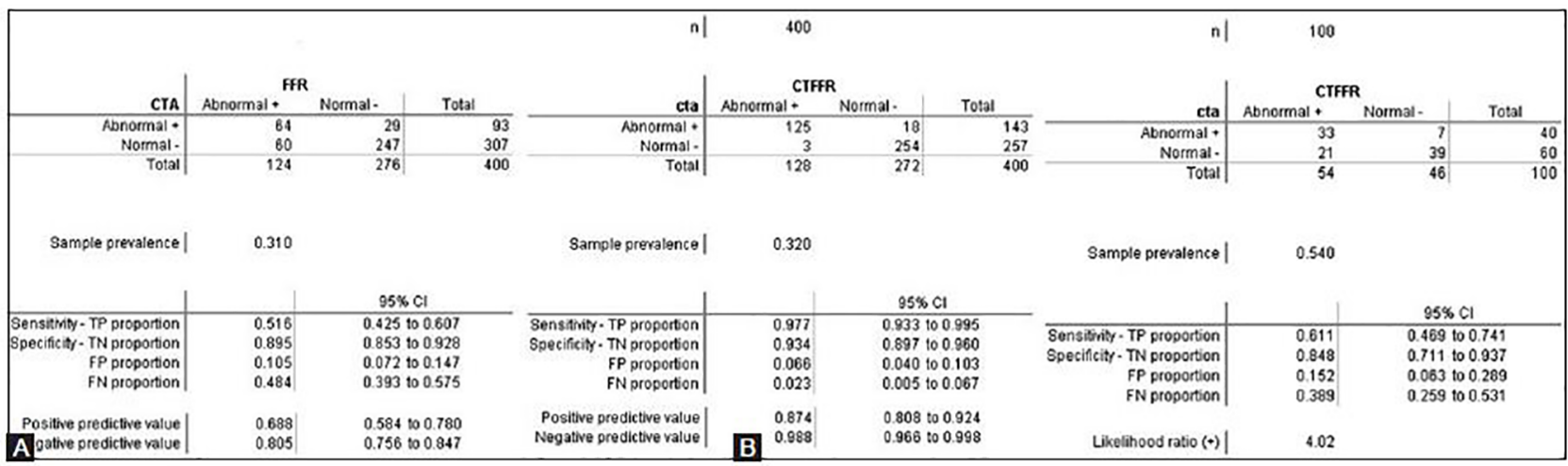

Fig. 8 (A) Sensitivity and specificity of CTCA in severe and significant stenosis, respectively. (B) Sensitivity and specificity of CTCA, a per-patient basis in severe stenosis. CTCA, computed tomographic coronary angiography.

$84 \%$, respectively ( - Fig. $\mathbf{8 B}$ ). There were $7 \%$ patients in the study who had either heavily calcified vessels where CTCA could not assess the lumen and stenosis due to blooming artifacts of calcium ( - Fig. 9A and B ). Eighteen vessels were calcified and were falsely labeled as stenotic on CTA, while three vessels were falsely labeled as normal but were positive 


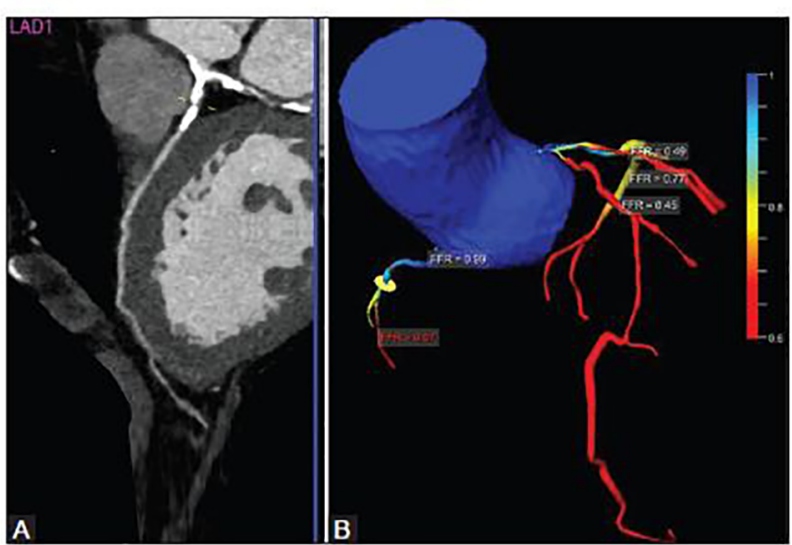

Fig. 9 (A) CTCA showing heavily calcified lumen of left anterior descending, left circumflex and left main coronary arteries. (B) CTFFR showing normal FFR in left main with ischemia in proximal left anterior descending and circumflex arteries. CTCA, computed tomographic coronary angiography; CTFFR, computed tomography fractional flow reserve.

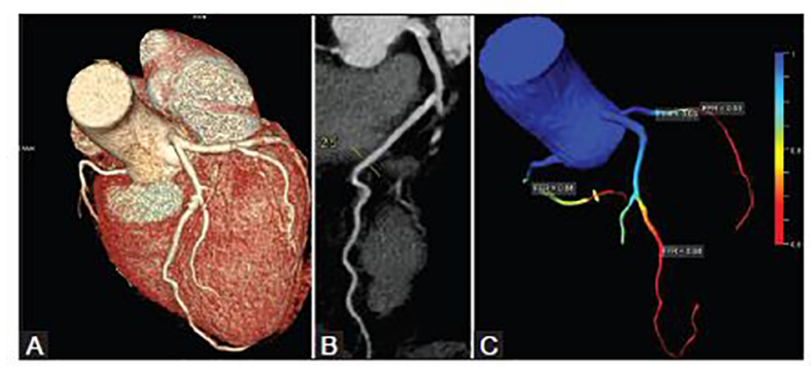

Fig. 10 (A) VRT image showing normally opacified left anterior descending artery. (B) Multiplanar reconstruction of left anterior descending artery with absent stenosis. (C) FFRCT showing nonobstructive coronary ischemia in mid segment with FFR of 0.64. CTFFR, computed tomography fractional flow reserve.

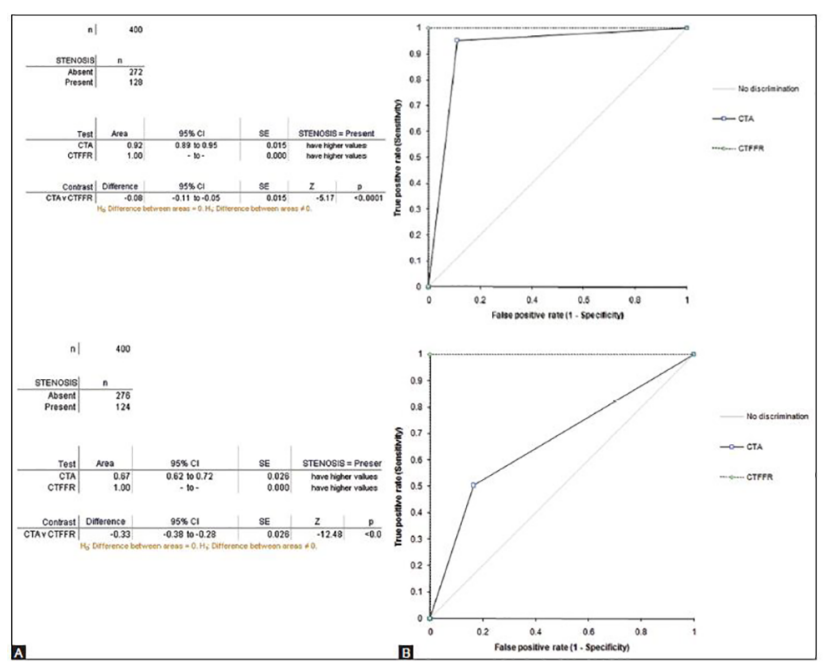

Fig. 11 (A) Area under curve for CTCA in detection of significant coronary stenosis per vessel basis. (B) Area under curve for CTA in severe coronary stenosis on per vessel basis. CTA, computed tomography angiography; CTCA, computed tomographic coronary angiography.
Table 2 Impact on Management plan after use of CTA and CTCA + CTFFR (fl/=100)

\begin{tabular}{|l|l|l|l|l|}
\hline SI. No modality & OMT & INVCA & PCI & CABG \\
\hline 1 Non invasive & 45 & 55 & nil & nil \\
\hline 2 CTCA & 67 & 33 & 12 & 11 \\
\hline 3 CTCA + CTFFR & 34 & 66 & 33 & 23 \\
\hline
\end{tabular}

Abbreviations: CABG: coronary artery bye pass graft; CTCA, computed tomographic coronary angiography; CTFFR, computed tomography fractional flow reserve; INVCA: Invasive coronary angiogram; OMT: optimal medical management; $\mathrm{PCl}$ : percutaneous coronary intervention.

on CTFFR ( - Fig. 10A-C). The AUC showed values of 0.92 and 0.67 for CTCA in detection of significant and severe stenosis, respectively, compared with CTFFR $(p<0.0001$; - Figs. 11A and $\mathbf{B}$ ). The results from questionnaires from treating clinicians showed that the use of CTFFR reclassified management of $21 \%$ patients with hemodynamic significant stenosis from optimal medical management to percutaneous interventions (PCI) and in $12 \%$ of patients from PCI to bypass grafting

- Table 2.

\section{Discussion}

This study shows that it is possible to calculate FFR noninvasively on site using CTFFR software based on imaging data of CTCA acquired with proper technique and modern state of art scanners in not more than 10 to 15 minutes which was the first prime objective of study. CTFFR accurately depicted the site of hemodynamically significant stenosis on colored FFR flow maps of the coronary tree map and also quantified FFR at the sites identified by the user. CTCA alone was accurate in identifying significant stenosis on per vessel basis with sensitivity and specificity of 97.7 and $93 \%$, respectively, with a negative predictive value of $98 \%$ which was very good to confirm the diagnosis. However, CTCA showed a reduced sensitivity of $51.6 \%$ for detection of severe stenosis with negative predictive value of $68.8 \%$ which meant that used alone it cannot plan revascularization procedures in such patients. On per patient basis, sensitivity and specificity of CTCA for severe stenosis were 61 and $84 \%$, respectively, which made $21 \%$ difference leading to misclassification of the management protocol based on CTCA alone. Our study also showed that the maximum difference was seen in patients with intermediate stenosis which on CTFFR was reclassified as hemodynamically significant or functionally severe. Ihdayhid et $\mathrm{a}^{11}$ also showed in their study that addition of FFRCT improved AUC of CTCA from 0.78 to 0.93 in the detection of severe stenosis and was even better than using CT myocardial perfusion which had AUC of 0.87 similar to what is seen in the present study. This has a major implication on the selection of patient management protocol as use of FFRCT increases the rate of percutaneous angioplasties in these patients who had discordance between anatomical and functionally significant stenosis. The use of CTFFR also modified the selection of vessels in $12 \%$ patients in the present study which changed the choice of treatment to bypass 
grafting. Similar results were shown by SYNTAX III trial ${ }^{12}$ in patients with three-vessel coronary artery disease; use of CTFFR changed treatment decisions and procedural planning in $20 \%$ patients and in $7 \%$ patients from angioplasty to surgery. There were $7 \%$ patients in the study who had either heavily calcified vessels where CTCA could not assess the lumen and stenosis due to blooming artifacts of calcium. Also, $2 \%$ patients in the study were falsely misclassified as severe stenosis due to similar effect. Although calcium scoring was not done by us as all patients who were enrolled in the study were symptomatic, these were false positive or not assessable on CTCA. Study shows that with FFRCT, these vessels could be evaluated and coronary calcium did not impair the functional evaluation as was seen in the determination of anatomic stenosis. NXT trial ${ }^{13}$ also showed that sensitivity of ischemia detection was high irrespective of coronary calcium scores and the Agatston scores up till 3,500 did not affect the results. There were three patients with ischemia in nonobstructive coronary arteries (INOCA) in our study, all of which were females in the study which is now emerging as an important clinical entity and has important implications on the prognosis of such patients. ${ }^{14}$

\section{Conclusion}

To conclude, the present study shows that use of on-site CTFFR is an accurate and quicker option not only to detect hemodynamically significant stenosis on CTCA but also to improve the sensitivity and specificity of noninvasive CT angiography which has a bearing on the patient management plan and treatment and can be frontline road map tool to evaluate such patients.

\section{Financial Support}

None.

\section{Conflicts of Interest}

There are no conflicts of interest.

\section{References}

1 Zhang LJ, Wu SY, Wang J, et al. Diagnostic accuracy of dual-source CT coronary angiography: The effect of average heart rate, heart rate variability, and calcium score in a clinical perspective. Acta Radiol 2010;51(7):727-740

2 National Institute for Health and Clinical Excellence, Chest Pain of Recent Onset: Assessment and Diagnosis of Recent Onset Chest Pain or Discomfort of Suspected Cardiac Origin. CG95. London, United Kingdom: National Institute for Health and Clinical Excellence; 2016
3 Douglas PS, Hoffmann U, Patel MR, et al. PROMISE Investigators. Outcomes of anatomical versus functional testing for coronary artery disease. N Engl J Med 2015;372(14):1291-1300

4 SCOT-HEART investigators CT coronary angiography in patients with suspected angina due to coronary artery disease (SCOT-HEART): An open-label, parallel group multicentre trial. Lancet 2015;285:2383-2391

5 Budoff MJ, Dowe D, Jollis JG, et al. Diagnostic performance of 64-multidetector row coronary computed tomographic angiography for evaluation of coronary artery stenosis in individuals without known coronary artery disease: results from the prospective multicenter ACCURACY (Assessment by Coronary Computed Tomographic Angiography of Individuals Undergoing Invasive Coronary Angiography) trial. J Am Coll Cardiol 2008;52(21):1724-1732

6 Pijls NH, De Bruyne B, Peels K, et al. Measurement of fractional flow reserve to assess the functional severity of coronary-artery stenoses. N Engl J Med 1996;334(26):1703-1708

7 De Bruyne B, Pijls NH, Kalesan B, et al; FAME 2 Trial Investigators. Fractional flow reserve-guided $\mathrm{PCI}$ versus medical therapy in stable coronary disease. N Engl J Med 2012;367(11):991-1001

8 Meijboom WB, Van Mieghem CA, van Pelt $\mathrm{N}$, et al. Comprehensive assessment of coronary artery stenoses: computed tomography coronary angiography versus conventional coronary angiography and correlation with fractional flow reserve in patients with stable angina. J Am Coll Cardiol 2008;52(8):636-643

9 Formaggia L Quarteroni A Veneziani A Cardiovascular Mathematics: Modeling and Simulation of the Circulatory System. Vol. 1. Heidelberg, Germany: Springer Science \& Buisness Media; 2010. . Murray CD. Proc Natl Acad Sci U S A 1926;12(3):207-214

10 Taylor CA, Fonte TA, Min JK. Computational fluid dynamics applied to cardiac computed tomography for noninvasive quantification of fractional flow reserve: scientific basis. J Am Coll Cardiol 2013;61(22):2233-2241

11 Ihdayhid AR, Sakaguchi T, Linde JJ, et al. Performance of computed tomography-derived fractional flow reserve using reduced-order modelling and static computed tomography stress myocardial perfusion imaging for detection of haemodynamically significant coronary stenosis. Eur Heart J Cardiovasc Imaging 2018;19(11):1234-1243

12 Andreini D, Modolo R, Katagiri Y, et al. SYNTAX III REVOLUTION Investigators. Impact of fractional flow reserve derived from coronary computed tomography angiography on heart team treatment decision-making in patients with multivessel coronary artery disease. Circ Cardiovasc Interv 2019;12(12):e007607

13 Nørgaard BL, Gaur S, Leipsic J, et al. Influence of coronary calcification on the diagnostic performance of CT angiography derived FFR in coronary artery disease: a substudy of the NXT trial. JACC Cardiovasc Imaging 2015;8(9):1045-1055

14 Pepine CJ, Ferdinand KC, Shaw LJ, et al. ACC CVD in Women Committee. Emergence of nonobstructive coronary artery disease: a woman's problem and need for change in definition on angiography. J Am Coll Cardiol 2015;66(17):1918-1933 